\title{
Increasing Commitment to Online Communities by Designing for Social Presence
}

\author{
Rosta Farzan, Laura Dabbish, Robert \\ Kraut \\ Carnegie Mellon University \\ rfarzan,dabbish,robert.kraut@cs.cmu.edu
}

\author{
Tom Postmes \\ University of Groningen \\ t.postmes@rug.nl
}

\begin{abstract}
The existence and survival of online communities depends upon the commitment and retention of their members. This paper compares alternative ways of designing online sites to increase member commitment. We report the results of two experiments conducted within a Facebook game application. The results show that designs that encourage relationships among members or emphasize the community as an entity both increase commitment and retention of players, but through different routes.
\end{abstract}

\section{Author Keywords}

Commitment, Online communities, Social attachment, Group identity, Facebook games

\section{ACM Classification Keywords}

H.5.3 Information Interfaces and Presentation: Group and Organization Interfaces: Evaluation/methodology, Web-based interaction.

\section{INTRODUCTION}

The social dimension of the Internet is clearly a major part of its attractiveness and success. According to Alexa.com, six of the top 10 sites in the world have a substantial social component, offering user-generated content (e.g., YouTube or Wikipedia) or supporting direct interaction among users (e.g., FaceBook or QQ). Despite their popularity, social sites experience high turnover, with most visitors coming only once and leaving after a short period. A recent survey found that most business efforts to build online communities failed, even when firms spent over $\$ 1$ million on the effort, primarily because of difficulties attracting people to the community and retaining them [25], [26].

Building commitment is a challenge even in the most successful online communities, such as Wikipedia, with more than three million articles and 1.5 million editors. Despite their success, users do not stick around. The modal number of edits on Wikipedia is one, and $60 \%$ of editors never re- turn after the first day of membership [14]. The Wikimedia foundation, the non-profit organization that runs Wikipedia, identified flagging commitment from editors and, more generally, "the health of the editing community as a major risk area for the Wikimedia movement."1

The existence and survival of online communities and their ability to provide resources to users depends upon the commitment level of their members. Lack of commitment can lead to conflict, lack of cooperation, decreased contribution and information sharing, higher rates of turnover and poor performance [7], [13]. Participants who feel greater commitment to an online community are more likely to provide the content that others value such as code in open source projects [5], or edits in Wikipedia [10]. Committed members care about and enforce norms of appropriate behavior. They are the ones who maintain the community and perform behind the scenes work to keep the online community going [2]. The challenge is that in virtual settings, people have difficulty developing commitment to the group or attachment to other members [6].

This paper compares alternative ways of designing online sites to increase members' commitment. It shows that designs that encourage relationships among members or that emphasize the community as an entity both increase commitment, but through different routes.

\section{Building Commitment}

In this paper, we are principally interested in the behavioral commitment of group members towards their group. According to social psychological research, people can become committed to a group or community in two distinct ways: (1) through group members' internalization of certain characteristics of the group as an entity (e.g., common interests, ethnicity, group history, norms and stereotypes, and/or competition with outgroups), which is the foundation for commitment to common identity groups, or (2) through interpersonal attractions among individual group members, which is the foundation for commitment to common bond groups [18], [20].

In principle, communities can be designed to enhance members' identity-based commitment or bond-based commitment,

\footnotetext{
${ }^{1}$ http://strategy.wikimedia.org/wiki/

Wikimedia_Foundation/Feb_2010_Letter_to_ the_Board
} 
supporting the representation of groups or individuals onscreen (e.g. [11], [16], [20]). For example, Wikipedia emphasizes common identity. It defines itself as "an online community of people interested in building a high-quality encyclopedia"2. It has policies that discourage interpersonal ties, a user interface that makes direct, private exchanges between community members difficult, and subgroups known as WikiProjects defined around common interests and interdependent tasks. In contrast, Facebook is more oriented towards common bonds. It promotes interpersonal ties among individuals and is based on interactions, news fields and exchange of pictures among Facebook "friends." However, Facebook also layers common identities via Facebook groups that are designed to connect users with a common interest.

Prior research in social psychology suggests that bond based and identity based attachments have distinct antecedents. Bond based attachment is rooted in interpersonal relations among individual group members. This form of attachment is driven by factors that increase interpersonal attraction such as interpersonal similarity, repeated exposure, communication and reciprocal self disclosure [1]. In contrast, identity-based commitment is derived from a connection to the group as an entity. People are more likely to identify with a group if it comprises a well-defined unit with common attributes and clear boundaries [3], [22], is given a common label, insignia or purpose, highlights homogeneity among members, suppresses information showing members as idiosyncratic individuals, and competes with out-groups [17].

Social psychological research also suggests that groups founded on common bonds and common identities may both elicit strong commitments, although not necessarily in identical ways. Specifically, common bond groups should display higher levels of interest in the individual group members and in within-group communications. In contrast, those in common identity groups should tend to treat individual group members as relatively interchangeable. Preserving homogeneity is a prerequisite for maintaining unity in such groups. For this reason, social psychological research often assumes that these two types of groups are antagonistic and cannot be combined with each other. It is argued that highlighting the presence of individuals should erode the common identity and highlighting the presence of the group as an entity should erode common bonds [21], [24], [23], [11], [16].

Much of the research on identity-based and bond-based commitment has used laboratory experiments to test predictions about the consequences of different types of group commitment. Because of the controlled nature of the psychological laboratory, it is not clear whether methods used to induce identity-based and bond-based commitment would be powerful enough to have behavioral effects in natural environments. Moreover, most social psychological experiments lack adequate measures of long-term behavioral commitment and focus instead on short-term psychological variables such as self-reported attachment and social influence.

\footnotetext{
${ }^{2}$ http://en.wikipedia.org/wiki/Wikipedia: What_Wikipedia_is_not
}

We are aware of only one study that attempted to induce identity based and bond-based attachment in online groups under realistic conditions [19]. This research tried to induce identity based attachment with an interaction design that emphasized subgroups through subgroup icons, frequently updated information about the subgroups, competition among subgroups, and group-oriented communication. With the identity-based design, information about individual members was suppressed (e.g., no member avatars). The research tried to induce bond-based attachment with a design that emphasized individuals (e.g., avatars representing individuals, repeated exposure to the same individuals, frequently updated information highlighting individual's behavior, and opportunities for pairwise communication). Results showed that both types of design increased commitment to an online movie database community, but identity-based attachment was easier to induce and more powerful in encouraging users to return.

While this prior research tested the effectiveness of common identity and common bond approaches to building commitment, it did not examine why the commitment occurs and the interaction of identity and common bond. Based on empirical research on antecedents of identity-based and bondbased attachment, we created designs of online groups that should lead to different types of attachment. The goal of the current research was not just to establish whether both designs would lead to greater commitment to the site (i.e., longer and more participation), but also to examine why. We expected that the bond-based designs - representing the presence of other individuals, repeated exposure to them, and opportunities for communication - would increase commitment through participants' attraction to the other group members, while identity-based designs - representing individuals at the group level, with distinct group name, presence of outgroups and competition among them - would increase commitment through identification with the group as an entity.

H1. Both common identity-based and common-bond-based community designs will induce behavioral commitment to the community, evidenced by longer and more participation in the group.

H2. Common bond-based community designs will induce behavioral commitment to the community through interpersonal attraction, evidenced by increased interest in the individuals in the community.

H3. Common identity-based community designs will induce behavioral commitment though identification with the group as an entity, evidenced by increased interest in intergroup comparisons.

The relationship between identity-based and bond-based commitment remains contested in the literature. Traditional assumptions maintain these two forms of group formation should not be mixed, and that attempting to combine identity based and bond based designs should be ineffective for commitment. However, there is correlational and experimental evidence suggesting there may be positive spill-over effects 
with common identities giving rise to the formation of interpersonal bonds [8] and interpersonal bonds forming the foundation for the emergence of shared identities [19], [15]. To explore this latter possibility, we include community designs that combine both bond-based and identity-based features, predicting that this combination would give rise to levels of commitment that are equally or more effective than either one in isolation.

H4. Bond-based and identity-based community designs may be combined, either maintaining or enhancing their effectiveness.

\section{RESEARCH SITE}

Facebook, the social networking site launched in 2004, has become the world's largest and most popular social website, with more than 400 million active users [4]. Facebook features an application platform which allows developers to implement different kinds of applications and integrate them into the site. Every month, more than $70 \%$ of Facebook users engage with the applications [4]. Games are among the most popular applications, attracting large number of users every day.

The popularity of Facebook games provided the opportunity to pursue our research among a large pool of users in a real setting. The abundance of Facebook applications decreases the time and attention users devote to each application. Increasing commitment to a single application has become an important challenge for the designers of those applications

We developed a Facebook application version of Tetris $\AA^{3}$ as the platform for our research. Tetris is a popular casual game. We chose Tetris as the focus task for our experiments, because it is typically solo and non-social. The game players are completely independent. This meant we could attribute any effects of our manipulations on social outcomes, such as increases in social attachment and commitment, to the designs we introduced.

Figure 1 presents the general design of the Tetris application interface in our study. The interface consists of five areas labeled in figure 1. Area 1 is the Tetris game itself, which is the classic version of Tetris. The objective of the game is to reach level 15. Players move up the levels after they clear 10-20 lines. Consistent with general game design paradigm, leveling up is easier in lower levels. As the levels increase, shapes fall more quickly, increasing the difficulty of forming lines. A single game can last between 2 to more than 20 minutes depending on skill levels of the player. The game area stayed the same in all experimental conditions. Area 2 is dedicated to presenting leader boards which show information about the players with the most achievements. The information inside the boards changed in each condition. Area 3 shows the name of the player or their team and the associated icon depending on the condition. To increase the general attractiveness of the game, we introduced weekly challenges. The challenges set a clear goal for players. According to goal setting theory, establishing a mea-

\footnotetext{
${ }^{3}$ http://www.tetrisfriends.com/
}

Table 1. Experimental Design of experiment 1

\begin{tabular}{llll}
\hline & \multicolumn{2}{c}{ Common Bond } \\
\cline { 3 - 4 } & & No & Yes \\
\hline $\begin{array}{l}\text { Common } \\
\text { Identity }\end{array}$ & No & $\begin{array}{l}\text { Self: control } \\
\text { group }\end{array}$ & $\begin{array}{l}\text { Within: inducing } \\
\text { bond based at- } \\
\text { tachment }\end{array}$ \\
& Yes & $\begin{array}{l}\text { Between: } \\
\text { inducing } \\
\text { identity based } \\
\text { attachment }\end{array}$ & $\begin{array}{l}\text { Within/Between: } \\
\text { bond and identity } \\
\text { based attachment } \\
\text { togther }\end{array}$ \\
\hline
\end{tabular}

surable discrete objective increases motivation and should therefore improve performance [12]. Area 4 shows the announcement of the current running challenge, the date the challenge is ending, and the current score to beat. The message is adopted to present the goal of each condition. Area 5 is dedicated to study manipulations. As described in more detail below, players in different conditions either saw information about their own prior games, information about the presence of other members of their team, information about the presence of other teams, or information about teammates and other teams.

\section{EXPERIMENT I - METHOD}

We designed our first experiment following the theoretical guidelines for inducing common bond and common identity. Table 1 presents the experimental design.

When players arrived at our application, in all experimental conditions they first had to choose a team to play in from a list of seven options (with each team identified by a name and an icon). Players in the non-social control condition went through a selection process as well, choosing an avatar before starting to play. The avatar icons were the same as the team icons.

We manipulated bond-based attachment by providing feedback about the presence of individuals within the team as well as opportunities for stylized communication with team members . Players in this condition competed with the other individuals in their team to have the highest score by the end of the week. The challenge standings included ranking, picture, name, and the top score for the top four players plus the participant. Next to the name of each player there was a thumbs-up icon that players could click to cheer each other on (figure 2(b)). The leader-boards in this condition featured information about the individual players in the team. We refer to this condition as "within" in the rest of the paper.

We manipulated identity based attachment by providing between team feedback about the player's own team as an entity in competition with other teams. Players in this condition were encouraged to score high to help their team win against other teams. The challenge standings in this condition included information about the top four teams plus the current player's team, including ranking, name, top score, and the icon of each team. No information about individuals 


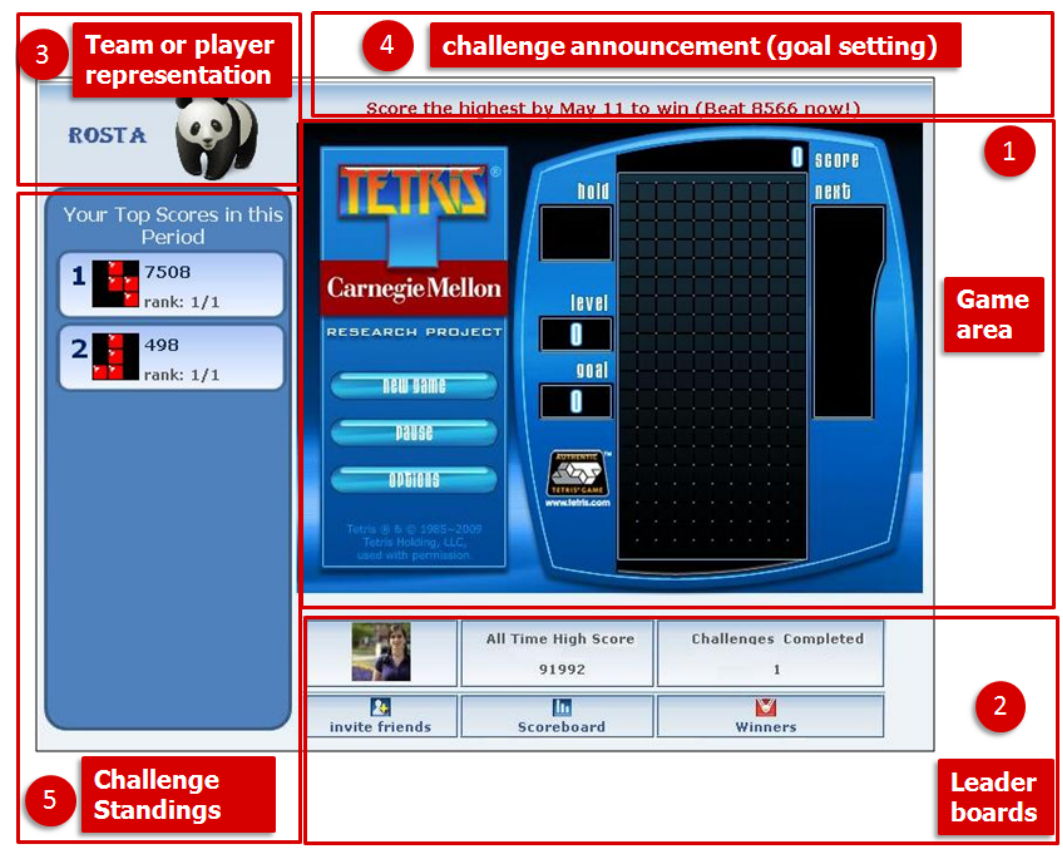

Figure 1. General design of research site

in the team was presented. Similar to the "within" condition, a thumbs-up icon next to the name of each team could be used to cheer on a team (figure 2(c)). The leader-boards in this condition featured information about teams. We refer to this condition as "between" in the rest of the paper.

We included a "within/between" condition to examine the interaction between a design encouraging identity-based attachment and interpersonal relationships among the individuals. The interface is shown in figure 2(d). Players in this condition were prompted to score high against their teammates and also to help their team win against other teams. One challenge standings displayed top teammates, while a second one displayed top teams. Players had the option to cheer individuals and/or teams. The leader-boards in this condition featured combined information about the individual players and the teams. We refer to this condition as "within/between" in the rest of the paper.

Our control condition was designed to include challenge elements with no social presence. Players were presented with a challenge score to beat. Information about the player's last seven games in the challenge period was displayed. The game history was ordered by score with the highest on the top (figure 2(a)). To control for the potentially motivating influence of constantly updating and increasing scores in social-challenges, the score to beat changed during the period challenge period, and to win players had to score higher than the last goal score at the end of the challenge. To set realistic goal scores which were comparable to other conditions, we presented players in the control condition with randomly selected high scores achieved in the "within" condition; The goal score for "control" conditions was the same as the highest score in a real team from that condition. Cheer- ing was not an option in this condition because there were no others present, so there was no cheer board. We refer to this condition as "self" in the rest of the paper.

\section{EXPERIMENT I - RESULTS}

We collected data from users joining the application between March 09, 2010 and March 25, 2010 . A total of 931 unique users joined the site in this time period. We tracked those users in the application until April 22, 2010. ${ }^{4}$ They were randomly assigned to one of the four conditions in round robin order. We collected counts and timestamps of all players' game actions such as game start and their visits to any leader-boards.

\section{Dependent Variables}

We assessed the effect of our manipulations on (1) commitment to the site and (2) social engagement. To measure commitment we examined survival and number of sessions played. Survival analysis shows how likely players are to come back and play over the days following their joining day. Total number of sessions played was another measure of commitment. We defined a session as continuous play with less than 15 minutes break between actions in the game. To measure social engagement, we analyzed how many times leader-boards were viewed. Viewing of leaderboards is a measure of curiosity about the actions of other players and teams.

\section{Statistical Analysis}

Number of sessions and views are count data and so are not normally distributed and they are overdispersed due to in-

\footnotetext{
${ }^{4}$ Due to some technical issues new users were not able to join after March 25.
} 


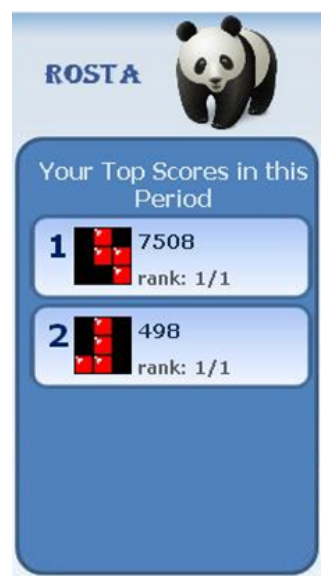

(a) self condition

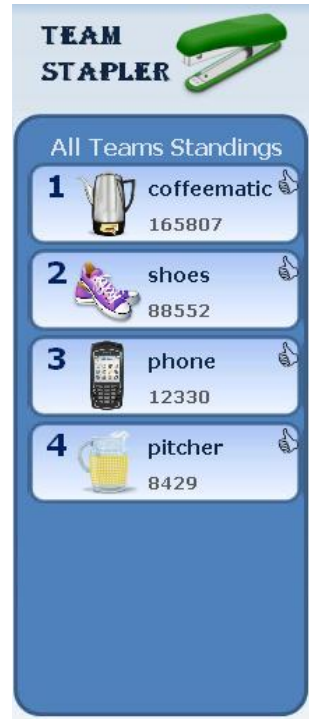

(c) between condition

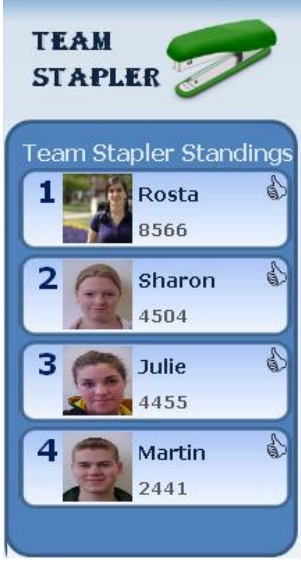

(b) within condition

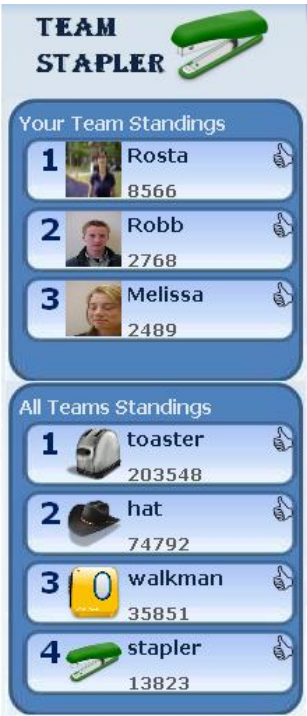

(d) within/between condition

Figure 2. Interface of different experimental conditions

flated occurrences of zero or one. We fitted negative binomial regression to predict the effect of conditions on these counts. The significance levels of all pairwise comparisons were adjusted using Bonferroni correction.

\section{Survival Analysis}

The survival analysis provides information about the fraction of the population in each condition that will survive past a certain time and the failure (dropout) rate for players in each condition. In this analysis, we defined the time intervals as days. The failure event is defined as the last day they played any game. We applied right censoring to players who played any time three days prior to the end of the experiment. Kaplan-Meier was used to estimate the survival function. Kaplan-Meier is a non-parametric estimation appropriate for data with non-normal distributions and takes into account missing data specified through right censoring [9].
The survival curves are presented in figure 3 and the analysis results are shown in table 2. The results show that assigning players to a group significantly increases the survival rate and the chance of returning, but there are no significant differences between the three 'social' conditions. After 10 days, only $7 \%$ of players in "self" condition continued playing on the site, while at least $16 \%$ of players in the experimental conditions remained.

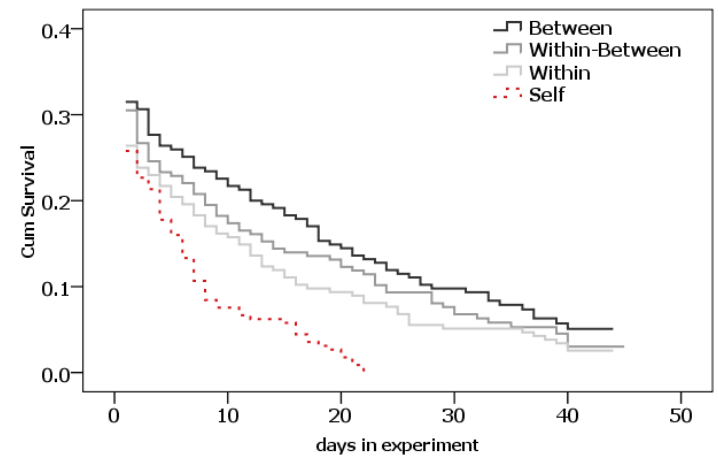

Figure 3. Experiment 1: Survival Analysis

Table 2. Mean Survival Time

\begin{tabular}{llll}
\hline & $\mathrm{N}$ & Mean & SE \\
\hline Self & 225 & $2.99^{\mathrm{a}}$ & .31 \\
Within & 235 & $5.33^{\mathrm{b}}$ & .66 \\
Between & 235 & $7.39^{\mathrm{b}}$ & .81 \\
Within/Between & 236 & $6.27^{\mathrm{b}}$ & .34 \\
\hline
\end{tabular}

$\dagger$ Different superscript $(\mathrm{a}, \mathrm{b})$ in the same column indicate significant differences between values $(\mathrm{p}<0.05)$

\section{Number of Sessions}

Our second measure of commitment was the average number of sessions played in each condition. Results are shown in figure 4. Similar to survival rate, assigning users to groups significantly increased the number of sessions played by $55 \%$, from an average of 2.41 to an average of 3.74 . There were no significant differences between experimental conditions.

\section{Viewing of Leader-boards}

In all conditions, participants could view a list of players with the highest score (scoreboard), and a list of players who won the most challenges (winnerboard). Challenge winners were players or teams (depending on the condition) with the highest score at the end of each challenge period. To simplify presentation and analysis of the results, we combined views of the scoreboard and winnerboard which were available in all four conditions. In the experimental conditions, the players could also view a list of players with the highest number of cheers (cheerboard). Higher attachment to the group as an entity should lead to higher interest in viewing the team-level boards, while interpersonal attraction should lead to higher interest viewing individual-level boards. The average number of views of each type of information is presented in figure 5. The analysis shows that players checked information presented on the boards most 


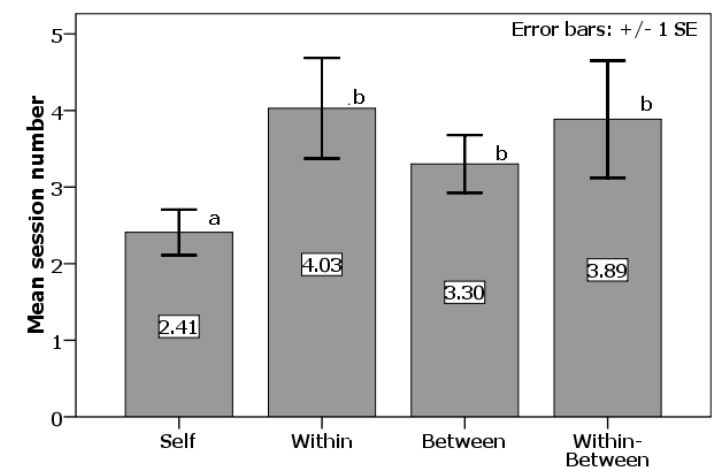

$\dagger$ Different superscript indicate significant differences between values $(\mathrm{p}<0.05)$

Figure 4. Experiment 1: Average number of session users played in each condition

often in the "within" condition, when individuals were represented. Representation of individuals along with teams ("within/between") had significantly higher social engagement compared with team information alone in the "between" condition.

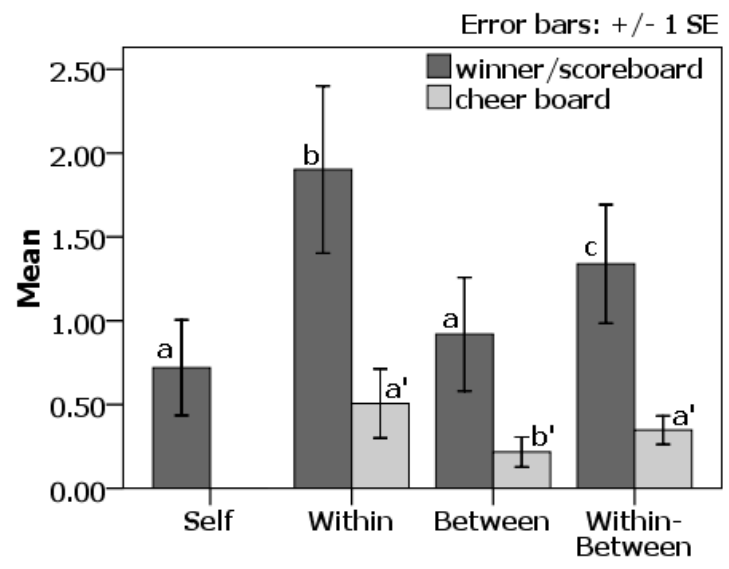

$\dagger$ Different superscript indicate significant differences between values $(\mathrm{p}<0.05)$

Figure 5. Experiment 1: Average number views of leader boards in each condition

\section{Mediation Analysis}

To evaluate our second and third hypothesis that bond versus identity-based community designs influence commitment throu different routes, we ran a mediation analysis. Viewing of leader boards is an indication of interest in group members (interpersonal attraction), or groups (intergroup comparisons); therefore, we treated viewing of leader boards as our mediating variable. We used number of sessions as our commitment measure (DV). To evaluate whether interpersonal attraction mediates commitment in the bond-based design condition ("within"), we ran mediation analysis comparing the "within" and "self" condition. To examine whether the same pathway mediated commitment in the identity-based condition, we ran the same analysis for "between" versus "self" condition. The mediation analysis path diagram is shown in figure 6. Path $c$ shows the indirect effect of condition on commitment which is significant in both cases. Path $a$ shows how the initial variable, condition, is correlated with the mediator, attraction, and path $b$ shows how the mediator affects the outcome variable, commitment. Path $c^{\prime}$ shows the direct effect of condition on commitment while the mediating effect of viewing leader-boards is taken into account. The results indicate that in the "within" condition, viewing of leader-boards completely mediates the effect of the design on commitment because there is no direct effect of condition on commitment once the mediation is considered. However, in the "between" condition, the direct effect remains marginally significant even after taking into account the mediation. The result supports our second hypothesis but only partially supports our third hypothesis.

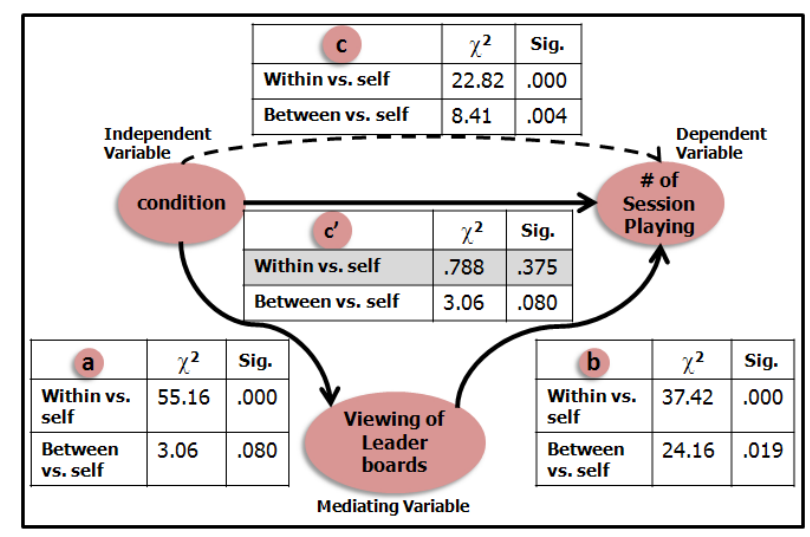

Figure 6. Experiment I: Mediation analysis

\section{EXPERIMENT I - CONCLUSION}

The results from this experiment provide evidence supporting our first hypothesis that both identity and bond-based attachment increases commitment. Presence of others as part of a team increases social engagement and curiosity about other players. Our second hypothesis was supported in that commitment as a result of bond-based design is mediated by interest in group members. The interest in teams partially mediates the commitment effect of common identity providing partial support for our third hypothesis. Our fourth hypothesis stated that common bond and identity designs can be integrated and would enhance each other. While we did not observe any significant enhancement effects in the "within/between" condition, our results do show that identity and bond-based designs can be combined so that they do h not cancel each other out-this condition has similar effects on players' commitments to the other experimental conditions. In terms of social engagement, adding individual level information increases players' interest in viewing leader-boards compared with team-only information.

Our first experiment has two limitations: (1) In the "self" condition, there was no representation of teams but additionally there was no competition with others - the goal is to improve personal score history. Therefore, we cannot distinguish the influence of connection to teams from presence of others and social competition. (2) The leader-boards in the "within/between" condition were not separated for teams 
versus individuals. Players had to click on the same set of button to access information either about teams or individuals. Therefore, we cannot distinguish identity versus bondbased social engagement and compare the combined condition with each isolated conditions.

\section{EXPERIMENT II - METHOD}

We designed experiment II to address the limitations of the first experiment. In order to address limitation (1), we added a new condition where players competed with everyone on the site but there were no team associations. We refer to this condition as "global" (see figure 7). In this condition, the challenge standings included the four top players on the site as a while plus the current player, but the players were not associated with any team. Because the leaderboard drew from a much larger pool (the entire site vs. seven team members), the turnover of individuals on the leaderboard was much higher than the "within" condition and players are not exposed to the same people repeatedly. We expected that bond-based attachment would not form as readily in this condition as in the "within" condition.

In order to clearly distinguish the effect of combining identity and bond-based attachment on social engagement, we separated individual leader boards from team leader boards as shown in figure 8. This allowed us to compare the interest in individual teammates with interest in other teams' as entities.

In addition to these experimental design changes, we also moved from classical version of Tetris to a two-minute version of the game where the objective is score as high as possible in two minutes. The change was a result of a usability study we conducted, showing that Facebook game players are more interested in low commitment short games. The game was changed across all conditions and resulted in higher rates of participation overall.

\section{EXPERIMENT II - RESULTS}

Experiment II ran from May 28, 2010 until June 25, 2010. A total of 3869 unique players joined the experiment during that time period and were randomly assigned to one of five conditions using a round robin procedure. The same dependent variables were measured in this experiment.

\section{Survival Analysis}

We applied the same procedure as experiment I to assess the survival rate of players in each condition. The survival curves are shown in figure 9. Contrary to experiment I, we did not observe any difference among the conditions and the chance of returning to the game stays the same for all conditions. This might be an artifact of change of the Tetris game into a more popular version of it.

\section{Number of Sessions}

We also examined commitment in terms of number of sessions played. The analysis of number of sessions played replicated the results of study I (figure 10). Players in "self" condition played a significantly lower number of sessions compared with all team conditions. However, players in the

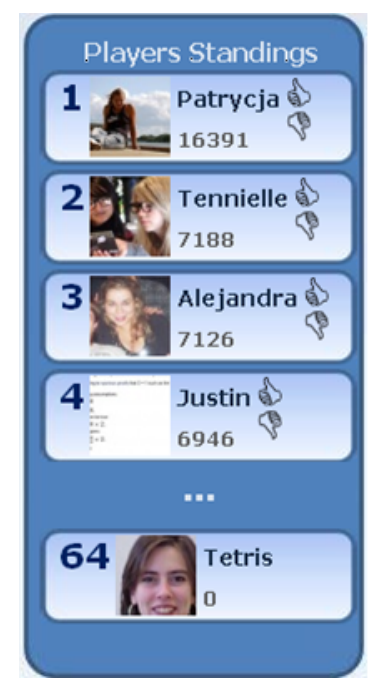

Figure 7. Global condition

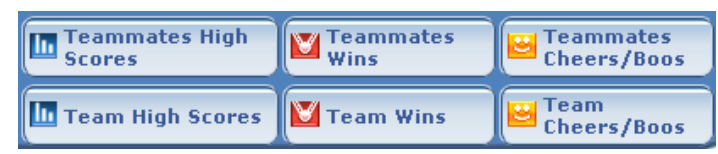

Figure 8. Team versus individual leader-boards

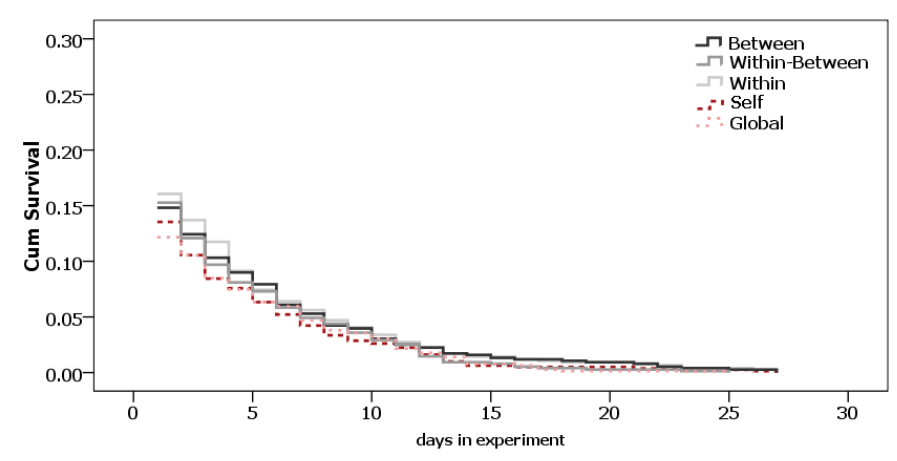

Figure 9. Experiment 2: Survival Analysis

"global" condition played a similar number of sessions as compared with experimental condition. This result suggests that mere presence of others increased liking of the game and led to a similar level of commitment as belonging to a team.

\section{Viewing of Leader-boards}

In experiment II we separated individual and team leader boards to be able to closely assess the effect of our manipulations on social engagement, measured by the number of leaderboard views. Similar to experiment I, players in the "control" condition were significantly less interested in viewing the leader boards as compared with "within" condition. Players in the "global" condition also viewed the boards significantly less than players in the "within" condition, suggesting that although the "global" condition did not differ from experimental conditions in terms of commit- 


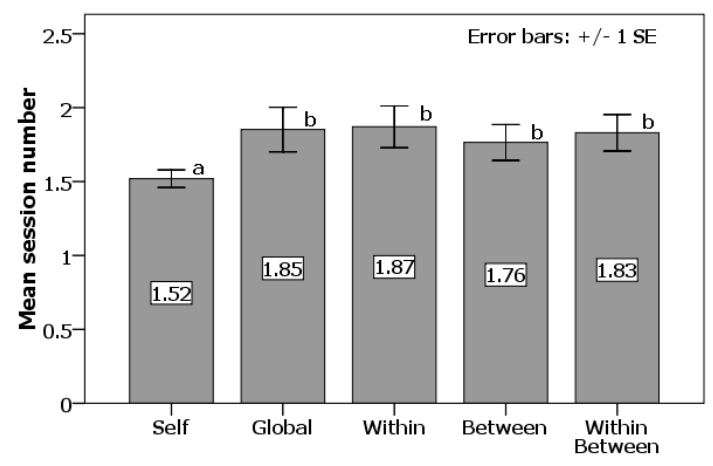

$\dagger$ Different superscript indicate significant differences between values $(\mathrm{p}<0.05)$

Figure 10. Experiment 2: Average number of session users played in each condition

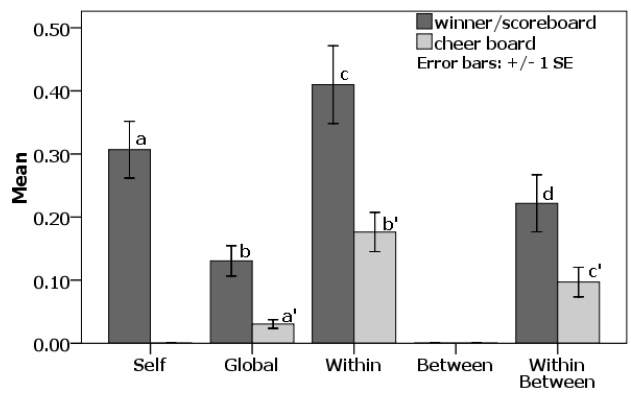

(a) Individual leader boards

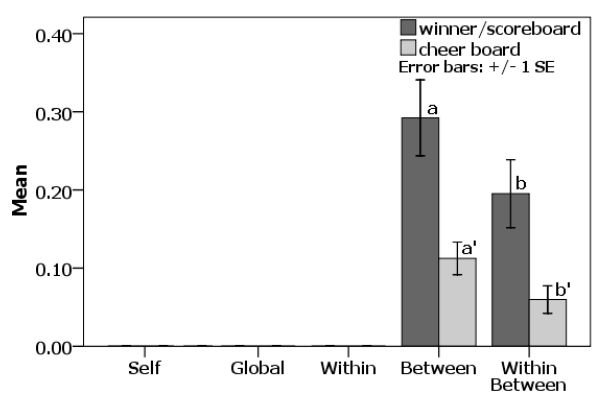

(b) Team leader boards

Figure 11. Average viewing of leader boards

ment, it differed in terms of social connection or interpersonal attraction. Players in the "within/between" condition viewed the individual leader boards significantly less than the "within" condition. This difference suggests that adding team-level information undercut players' interest in individuals.

On the other hand, comparison of team leader boards shows that players in the "within/between" condition viewed the team boards significantly less than in the "between" condition. The results hint that integration of individual and team level information could have caused information overload which decreased social engagement.

\section{Cheering}

Limited stylized communication was available in all experimental condition through cheering and booing of players and teams. Table 3 shows the average number of cheers and boos and the percentage of boos in each condition. Results suggest that the experimental conditions engendered a different type of social relationship among players than the "global" condition. Players in all three experimental conditions cheered significantly more than players in "global" condition. Players in the experimental conditions were also significantly more likely to cheer than boo others, while in the "global" condition, the players were equally likely to cheer and boo others. This result provides another indication that social engagement was lower in the "global" condition comparing with experimental conditions despite similar levels of commitment in terms of retention and game playing ${ }^{5}$.

Table 3. Cheering and Booing

\begin{tabular}{lccc}
\hline & \multicolumn{2}{l}{ Cheering \& Booing } & \% of Cheers \\
\cline { 2 - 3 } & Mean & SE & \\
\hline Global & .10 & .03 & $52 \%$ \\
Within & .20 & .03 & $74 \%$ \\
Between & .21 & .04 & $67 \%$ \\
Within/Between & .32 & .05 & $75 \%$ \\
\hline
\end{tabular}

\section{MEDIATION ANALYSIS}

Similar to experiment I, we conducted a mediation analysis to evaluate whether the experimental designs influenced commitment through social engagement. The path diagram is shown in figure 12. We found that social engagement in the form of leader-board viewing fully mediated the effect of the "within" condition on number of sessions played. This result supports our second hypothesis that bond-based designs influences commitment through their influence on interpersonal attraction. However, team leaderboard viewing did not mediate the effect of the "between" condition on commitment in terms of number of sessions played, providing no support for our third hypothesis. Additionally, the mediation analysis shows that in the "global" condition, viewing of leader-boards does not mediate the attachment effect. Different factors seem to drive commitment in the "global" than in the "within" condition. Even though similar information about individuals was presented on the leader boards in these conditions, lack of association with a welldefined team and repeated exposure to the same individuals resulted in lower attachment in terms of bonds and subsequently reduced interest in group members. In the "global" condition, presence of others and competition with them increased liking of the game, and therefore commitment, but did not create interpersonal relationships among players as in the "within" condition.

\section{Conclusions}

The results of experiment I were largely replicated. Presence of others and competition with them as teams or individuals

\footnotetext{
${ }^{5}$ The number of cheers did not differ among experimental conditions in experiment I. Due to lack of space and non-significant differences, we did not report that in the paper
} 


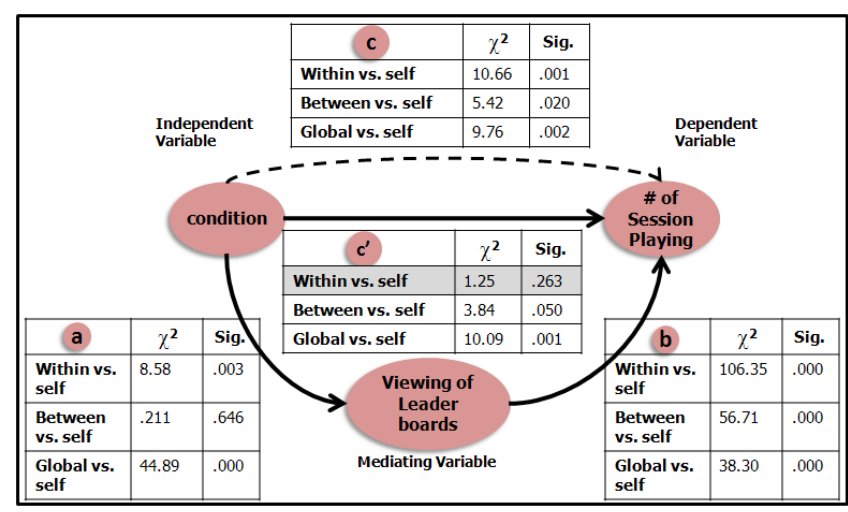

Figure 12. Experiment II: Mediation analysis

increased the number of game play sessions. By adding the "global" competition condition, we were able to distinguish the effect of attachment versus mere presence of others. Although both can increase behavioral commitment, they have different effects on players' behavior and seem to increase commitment through different paths. Presence of others increased behavioral commitment but did not affect social engagement. Being associated with a team led to liking people in the team and interpersonal attraction which encouraged returning to game.

\section{DISCUSSION AND FUTURE WORK}

The results of our studies support the idea that social presence of others can be manipulated on-screen to foster the formation of common bonds and common identities, and that this visual presence leads to greater commitment to the site and longer and more participation. We also showed that the subsequent attachment happens via different routes, showing evidence for the theoretical processes involved. In the bondbased condition, in which team association and repeated exposure to individuals are provided, commitment is mediated by interest in individual group members.

Contrary to traditional views on combining common bond and identity, we showed that the integration of the two does not undercut behavioral commitment. However, our straightforward way of combining these two designs also did not have enhancing effects. It may be that a more integrated display, which embedded individual images in team iconography or vice versa, would have a more enhancing effect. The combined design also may have overloaded players because of additional viewing options. Although the amount of total social engagement increased in the combined condition, the interest in individuals or teams alone was less than each comparable condition.

One of the features of the "global" condition in the second experiment was that top players were chosen from a large pool of players. As a result, the top players frequently changed as opposed to experimental conditions where top players were chosen from a team of seven players; i.e. players in experimental conditions were repeatedly exposed to the same people, especially during the same challenge pe- riod. This difference in repeated exposure could also have contributed to the lower social engagement in the "global" condition. In general, the "global" condition could be perceived as a team with a very large number of members (in contrast to the smaller teams in the experimental conditions). As part of our future work we are planning to investigate the effect of group size on inducing identity and bond based attachment.

Although team attachment and competition with others were successful in increasing commitment, we still observed low return rates and commitment in general. We believe increasing salience of teams and communication among team members will further improve attachment. We are planning on increasing team prominence by allowing the players more voice in the team selection process. Currently, communication among the players is limited to cheering or booing. Encouraging communication among a group of people who do not have prior contact is challenging. We are planning to investigate the effectiveness of different forms of stylized communications to increase levels of communication.

\section{DESIGN IMPLICATIONS}

In the current work, we were able to systematically vary onscreen representations of teams and individuals in a way that strongly affected behavioral commitment and social engagement, even in the context of a non-social task (a solo playing game). The design implication is that on-screen presence is a powerful cue for the formation of online communities, and that the nature of this cue triggers the formation of qualitatively different kinds of communities (common bond, common identity, and a hybrid form which combines elements of both). These different designs may map on to the different goals that online communities may seek to achieve.

Increasing return rate and commitment is the most important goal for most online communities-all manners of enhancing social presence seem to be suitable for this. However, a high degree of social engagement might not always be required and might even harm the utility of some communities by distracting members from their task-fostering the formation of common identities may guide designs here. On the other hand, communities who rely on members' interpersonal communication and involvement may require a high level of social engagement in addition to behavioral commitment. Health support communities are one example in which members are looking for strong support from other members-fostering formation of common bonds may guide design choices. However, in all communities, it appears that encouraging the formation of shared identities and common bonds, in isolation or combination, may help such communities increase the return rate of their members, either through fostering the formation of interpersonal relationships or through fostering attachment to the group as a whole.

\section{REFERENCES}

1. BERSCHEID, E., AND ReIS, H. T. Attraction and close relationships. In The Handbook of Social Psychology, D. T. Gilbert, S. T. Fiske, and G. Lindzey, Eds., vol. 2. New York: McGraw-Hill, 1998, pp. 193-281. 
2. Butler, B., Sproull, L., Kiesler, S., AND KRAUT, R. Community effort in online groups: Who does the work and why? Leadership at a distance 11 (2007), 171-194.

3. Castano, E. On the advantages of reifying the ingroup. In The psychology of group perception: Contributions to the study of homogeneity, entitativity and essentialism, V. Yzerbyt, C. M. Judd, and O. Corneille, Eds. New York: Psychology Press, 2004, pp. 381-400.

4. FACEBOOK. Press room - statistics. http://www.facebook.com/press/info.php?statistics, 2010.

5. Herbsleb, J. D., ANd Mockus, A. An empirical study of speed and communication in globally distributed software development. In IEEE Transactions on Software Engineering (2003), vol. 29, pp. 481-494.

6. Hertel, G., Geister, S., ANd Konradt, U. Managing virtual teams: A review of current emperical research. Human Resources Management Review 15, 1 (2005), 69-95.

7. Hinds, P. J., AND BAILEY, D. E. Out of sight, out of sync: Understanding conflict in distributed teams. Organization Science 14, 6 (2003), 615-632.

8. HoGG, M. A. The social psychology of group cohesiveness: From attraction to social identity. London: Harvester/Wheatsheaf, 1992.

9. Kaplan, E. L., AND Meier, P. Nonparametric estimation from incompelte observations. American Statistical Association 53, 282 (1958), 457-481.

10. Kittur, A., Suh, B., Pendelton, B. A., And Chi, E. H. He says, she says: Conflict and coordination in wikipedia. In CM Conference on Human Factors in Computing Systems (2007), ACM Press, pp. 453-462.

11. LEA, M., AND SPEARs, R. Computer-mediated communication, de-individuation and group decision-making. Special issue: Computer-supported cooperative work and groupware. International Journal of Man Machine Studies 34 (1991), 283-301.

12. Locke, E. A., AND Latham, G. P. Building a practically useful theory of goal setting and task motivation. American Psychologist 57 (2002), 705-717.

13. Olson, G. M., AND Olson, J. S. Distance matters. Human Computer Interaction 15 (2000), 139-178.

14. Pansiera, K., Halfaker, A., And Terveen, L. Wikipedians are born, not made. In ACM Special Interest Group on Computer-Human Interaction (2009).
15. Postmes, T., And Spears, R. Individuality and social influence in groups: Inductive and deductive routes to group identity. Personality and Social Psychology 89, 5 (2005), 747-763.

16. Postmes, T., Spears, R., And Lea, M. Breaching or building social boundaries? side-effects of computer-mediated communication. Communication Research 25 (1998), 689-715.

17. Postmes, T., Spears, R., Sakhel, K., And DE GROOT, D. Social influence in computer-mediated communication: The effects of anonymity on group behavior. Personality and Social Psychology Bulletin 27 (2001), 1243-1254.

18. Prentice, D. A., Miller, D. T., And Lightdale, J. R. Asymmetries in attachments to groups and to their members: Distinguishing between common-identity and common-bond groups. Personality and Social Psychology Bulletin 20, 5 (1994), 484-493.

19. Ren, Y., Harper, F. M., Drenner, S., Terveen, L., Kiesler, S., Riedl, J., AND KraUt, R. Increasing attachment to online communities: Evidence-based design. MIS Quarterly (Under review).

20. Ren, Y., Kraut, R., AND Kiesler, S. Applying common identity and bond theory to design of online communities. Organization Studies 28, 3 (2007), 377-408.

21. Sassenberg, K. Common bond and common identity groups on the internet: Attachment and normative behavior in on-topic and off-topic chats. Group Dynamics: Theory and Practice 6, 1 (2002), 27-37.

22. Sherman, S., Hamilton, D., And Lewis, A. Perceived entitativity and the social identity value of group memberships. In Social identity and social cognition, D. Abrams and M. A. Hogg, Eds. Malden, MA: Blackwell, 1999, pp. 80-110.

23. TURner, J. C. Social influence. Uk: Open University Press (1991).

24. Wilder, D. A., AND Simon, A. F. Categorical and dynamic groups: Implications for social perception and intergroup behavior. In Intergroup cognition and intergroup behavior, C. Sedikides, J. Schopler, and C. A. Insko, Eds. Mahwah, NJ: Erlbaum, 1998, pp. 27-44.

25. WORTHEN, B. Why most online communities fail. Wall Street Journal (2008).

26. YoOn, J., BAKER, M., AND Ko, J. Interpersonal attachment and organizational commitment: Subgroup hypothesis revisited. Human Relations 47, 3 (1994), 329-351. 